\title{
Patient Selection and Timing of Continuous Renal Replacement Therapy
}

\author{
Marlies Ostermann ${ }^{a}$ Michael Joannidis $^{b}$ Antonello Pani $^{c}$ Matteo Floris ${ }^{c}$ \\ Silvia De Rosa ${ }^{d}$ John A. Kellum ${ }^{e}$ Claudio Ronco $^{d}$ on behalf of the 17 th Acute \\ Disease Quality Initiative (ADQI) Consensus Group
}

${ }^{a}$ Department of Nephrology and Critical Care, Guy's and St. Thomas' Hospital, London, UK; ${ }^{b}$ Division of Intensive Care and Emergency Medicine, Department of Internal Medicine, Medical University Innsbruck, Innsbruck, Austria; 'Renal Unit, Ospedale 'G. Brotzu', Cagliari, and 'Department of Nephrology, Dialysis and Transplantation, International Renal Research Institute of Vicenza, San Bortolo Hospital, Vicenza, Italy; ${ }^{\circ}$ Center for Critical Care Nephrology, CRISMA, Department of Critical Care Medicine, University of Pittsburgh School of Medicine, Pittsburgh, Pa., USA

\section{Key Words}

Acute kidney injury . Continuous renal replacement therapy $\cdot$ Precision medicine $\cdot$ Hemodialysis

\section{Abstract \\ When and in whom to initiate continuous renal replacement therapy (CRRT) for acute kidney injury (AKI) remains a highly controversial topic with large practice variation around the world. Even within countries, practice variation exists and}

Faculty of the 17th ADQI International Consensus Conference: Bagshaw S.M., Critical Care Medicine, Faculty of Medicine and Dentistry, University of Alberta, Edmonton, Canada; Chakravarthi R.M., Department of Nephrology, STAR Hospitals, Hyderabad, India; De Rosa S., International Renal Research Institute of Vicenza (IRRIV), San Bortolo Hospital, Vicenza, Italy; Floris M., Renal Unit, Ospedale 'G. Brotzu', Cagliari, Italy; Hoste E.A.J., Ghent University Hospital, Ghent University, Ghent, Belgium; Research Foundation Flanders, Brussels, Belgium; Joannidis M., Division of Intensive Care and Emergency Medicine, Department of Internal Medicine, Medical University Innsbruck, Innsbruck, Austria; Mehta R.L., University of California San Diego, San Diego, USA; Murugan R., Department of Critical Care Medicine, Center for Critical Care Nephrology, University of Pittsburgh School of Medicine, Pittsburgh, USA; Neri M., International Renal Research Institute of Vicenza (IRRIV), San Bortolo Hospital, Vicenza, Italy; Pani A., recommendations for clinical practice are not specific. In this article, we report the consensus recommendations for timing and patient selection for CRRT - the results of the 2016 Acute Disease Quality Initiative XVII conference on 'precision CRRT'. We suggest that these recommendations could serve to develop the best clinical practice and standards of care for use of CRRT in patients with AKI. Finally, we identify and highlight the areas of ongoing uncertainty and propose an agenda for future research.

c) 2016 S. Karger AG, Basel

Renal Unit, Ospedale 'G. Brotzu', Cagliari, Italy; Ostermann M., Guy's \& St. Thomas' Hospital, London, UK; Ricci Z., Department of Pediatric Cardiology and Cardiac Surgery, Ospedale Pediatrico Bambino Gesù IRCCS, Roma, Italy; Rosner M., Department of Medicine, University of Virginia, Charlottesville, USA; Samoni S., Institute of Life Sciences, Sant'Anna School of Advanced Studies, Pisa, Italy; Tolwani A.J., Department of Nephrology, University of Alabama at Birmingham, USA; Xiaoqiang Ding, Department of Nephrology, Shanghai Institute of Kidney and Dialysis, Zhongshan Hospital, Fudan University, Shanghai, China; Wuhua Jiang, Department of Nephrology, Shanghai Institute of Kidney and Dialysis, Zhongshan Hospital, Fudan University, Shanghai, China.

Report from the 17th Acute Disease Quality Initiative (ADQI) Consensus Conference. Asiago, Italy, June 10-13, 2016.

\section{KARGER}

E-Mail karger@karger.com

www.karger.com/bpu
C 2016 S. Karger AG, Basel

0253-5068/16/0423-0224\$39.50/0
Prof. John A. Kellum, MD

Department of Critical Care Medicine

Center for Critical Care Nephrology, University of Pittsburgh School of Medicine 604 Scaife Hall, 3550 Terrace Street Pittsburgh, PA 15261 (USA)

E-Mail kelllum@pitt.edu 


\section{Introduction}

Practice patterns for continuous renal replacement therapy (CRRT) are extremely variable. Broadly speaking, CRRT is almost exclusively applied to patients in the intensive care unit (ICU). However, 2 patient-level epidemiologic studies $[1,2]$, surveys of self-reported practice patterns $[3,4]$ and several large case series $[5,6]$ have documented large variation in clinical practice. In 1996, Mehta and Letteri [3] surveyed 2,000 nephrologists in the US and found that less than $25 \%$ of patients with acute renal failure were treated with CRRT. The use of CRRT is much more common in Europe, although its use is highly variable between centers, while CRRT is the predominant $(>90 \%)$ choice in Australia $[2,4]$.

In general, it appears that the decision to use CRRT is affected by strongly held physician beliefs as well as a number of patient and organizational characteristics. Patient characteristics may include age, gender, race, illness acuity and comorbidities. Organizational characteristics vary depending on country, type of institution, type of ICU, type of physician or insurance provider and perceived cost of therapy. However, the strength of association of these characteristics with the decision to use CRRT is not fully understood. Furthermore, large epidemiological studies are needed to establish the factors that are most important in determining practice patterns, and whether there are important access-to-care issues.

\section{Methods}

The Acute Disease Quality Initiative (ADQI) process has been described previously [7].

Briefly, we convened a 3-day consensus conference in Asiago, Italy, in June 2016, and invited experts in nephrology and critical care medicine from both adult medicine and pediatrics. Principles of current practice were debated in light of existing evidence. Areas of broad consensus were described and a research agenda was developed using a modified Delphi method. Prior to the conference, we identified fundamental questions for which consensus would be sought. During the pre-conference phase, each workgroup performed comprehensive literature searches to summarize existing knowledge and to identify gaps that could be addressed by future research. Core questions/concepts were crafted and these were presented to the entire ADQI consensus group during the conference; a series of breakout sessions and plenary presentations allowed debate, discussion and refinement of these concepts. The 'timing and patient selection workgroup' performed a literature search using the following terms: 'renal replacement therapy' together with either 'acute kidney injury', 'acute renal failure' or 'continuous veno-venous hemodialysis', 'continuous veno-venous hemofiltration', 'hemodialysis', 'SLED', 'ultrafiltration' or 'extra-

Timing and Patient Selection for CRRT
Table 1. Questions addressed

(1) When should acute RRT be initiated? (includes AKI and non-AKI indications)

(2) What is the most appropriate therapy to meet a demandcapacity imbalance for a specific patient?

(3) How should RRT be integrated into other extracorporeal therapies?

(4) When should transition of modalities be considered (CRRT, IRRT, hybrid therapy)?

(5) How should patients be liberated from RRT?

corporeal' in MEDLINE using PubMed as the search engine. The 5 core questions ultimately addressed by the workgroup are shown in table 1.

\section{Results}

When Should Acute Renal Replacement Therapy Be

\section{Initiated?}

Consensus statement 1.1: Acute RRT should be considered when metabolic and fluid demands exceed total kidney capacity.

Over the last 2 decades, widely accepted definitions of acute kidney injury (AKI) have evolved from the Risk, Injury, Failure, Loss and End-stage renal disease criteria to the AKI Network classification and the Kidney Disease Improving Global Outcomes (KDIGO) criteria. All are based on acute changes in serum creatinine ( $\mathrm{SCr}$ ) and/or a fall in urinary output. They include 3 stages of severity of AKI. While AKI stage correlates with both hospital and 1-year mortality, it is not an indication for RRT per se. In fact, many patients with even stage $3 \mathrm{AKI}$ will have spontaneous recovery without RRT. In addition, some patients may have urgent indications for RRT (e.g., hyperkalemia, fluid overload) without meeting criteria for even stage 2 AKI. Numerous factors should be considered when considering initiation of RRT for AKI (table 2).

Until recently, there were only a few small randomized controlled trials (RCTs) examining timing of initiation of RRT but numerous observational and cohort studies [8, 9]. Most observational studies concluded that patients receiving RRT had a better outcome if RRT was provided earlier. However, the risk of bias is high in these studies since they did not include a control group without RRT. Also, a proportion of patients (especially those in the 'ear- 
Table 2. Factors to consider for RRT initiation*

Severity of illness and trajectory

AKI severity and trend

Severity of electrolyte and acid base disorder

Fluid balance and symptoms of fluid overload

Presence of relevant organ dysfunction impacted by AKI/fluid overload

Necessity of RRT

Likelihood of early recovery of kidney function without RRT

Underlying comorbidities impacted by AKI/fluid overload

Associated acute organ dysfunction

Risks of RRT

Vascular access

Hemodynamic instability

Infection

Clearance of trace elements/water soluble vitamins/drugs

Immobilization

Other factors

Patient and family wishes

Overall goals of care

Availability of machines and nursing staff

Healthcare costs

* Adapted from Macedo and Mehta [18].

ly RRT' group) may have received RRT unnecessarily. A recent retrospective study included a control group without RRT and showed that after propensity score matching, patients in the RRT group had a higher mortality, especially those with less severe AKI [10]. Since the majority of patients with AKI never receive RRT in clinical practice, existing studies and analyses provide insufficient guidance on those who should receive RRT. A recent analysis using propensity matching concluded that patients with AKI only clearly benefitted from RRT if they developed severe azotemia ( $\mathrm{SCr}>4.2 \mathrm{mg} / \mathrm{dl}$ ) [11]. Clearly this leaves the conundrum that patients who are likely to benefit most from RRT are not easy to identify early at a stage when they may benefit most.

In 2015 and 2016, the results of 3 large RCTs were reported [12-14]. 'Early' and 'late' RRT was defined by the KDIGO criteria; 2 studies included an additional AKI biomarker (neutrophil gelatinase-associated lipocalin $(\mathrm{NGAL}))[12,13]$. Wald et al. [12] performed a feasibility study in 100 patients with AKI stage 2 and whole blood NGAL $\geq 400 \mathrm{ng} / \mathrm{ml}$. Patients had a high severity of illness (Sequential Organ Failure Assessment (SOFA) score [14]) and $\sim 90 \%$ were treated with mechanical ventilation and vasopressors. In the 'accelerated' arm, RRT was com- menced within $12 \mathrm{~h}$ of meeting the inclusion criteria, whereas in the 'standard' arm, initiation of RRT was determined by the so called 'classic indications', that is, hyperkalemia, acidosis and severe respiratory failure. There was no difference in mortality between both groups. About $30 \%$ of patients randomized to 'standard' treatment did not require RRT. In contrast, in a single center study, Zarbock et al. [13] randomized 231 predominantly cardiac surgical patients with AKI stage 2 and a serum NGAL $>150 \mathrm{ng} / \mathrm{ml}$ to early versus late RRT. The average SOFA score was 16 . Mortality of patients randomized to early treatment as defined by RRT within $8 \mathrm{~h}$ of AKI stage 2 had a $35 \%$ lower mortality than patients randomized to late RRT, that is, within $12 \mathrm{~h}$ of reaching AKI stage 3. Only $10 \%$ of patients in the late group did not require RRT. To date, the largest multicenter RCT investigating timing of RRT included 620 critically ill patients with an average SOFA score of 11 [14]. The predominant first treatment modality was intermittent hemodialysis (IHD, 55\%). Patients randomized to 'early' treatment received RRT within $6 \mathrm{~h}$ of reaching AKI stage 3 whereas 'late' treatment was only initiated when 'absolute' indications were fulfilled, including hyperkalemia, acidemia, oliguria for $>72 \mathrm{~h}$, blood urea nitrogen $>110 \mathrm{mg} / \mathrm{dl}$ or pulmonary edema. Mortality was around 50\% with no significant difference between both groups. However, $60 \%$ of patients randomized to 'late' treatment did not require RRT. Importantly, patients in the 'late' treatment group who were started on RRT had the highest mortality (62\%) whereas mortality of patients who did not require RRT was even lower than that of patients in the 'early' RRT group.

There is a clear association between fluid overload and outcome but a causative link has not been confirmed [15]. In certain subgroups of patients, diuretics can be effective at preventing and correcting fluid overload (i.e., in patients with acute lung injury or congestive heart failure) [16] but RRT is often needed in critically ill patients with limited physiological reserve to tolerate fluid overload. This is particularly relevant in patients with cardiac and/ or respiratory problems.

Based on the existing evidence, the decision to start acute RRT should be individualized and not be based solely on renal function or stage of AKI without considering the clinical context $[17,18]$. It is assumed that the kidneys have a finite capacity. Consequently, the initiation of RRT should be prompted by the ability of the kidney to meet the demands being placed on them $[19,20]$. An increasing demand causes an imbalance and may lead to dysfunction in other organs unless corrected quickly. In situations where a significant gap between demand 


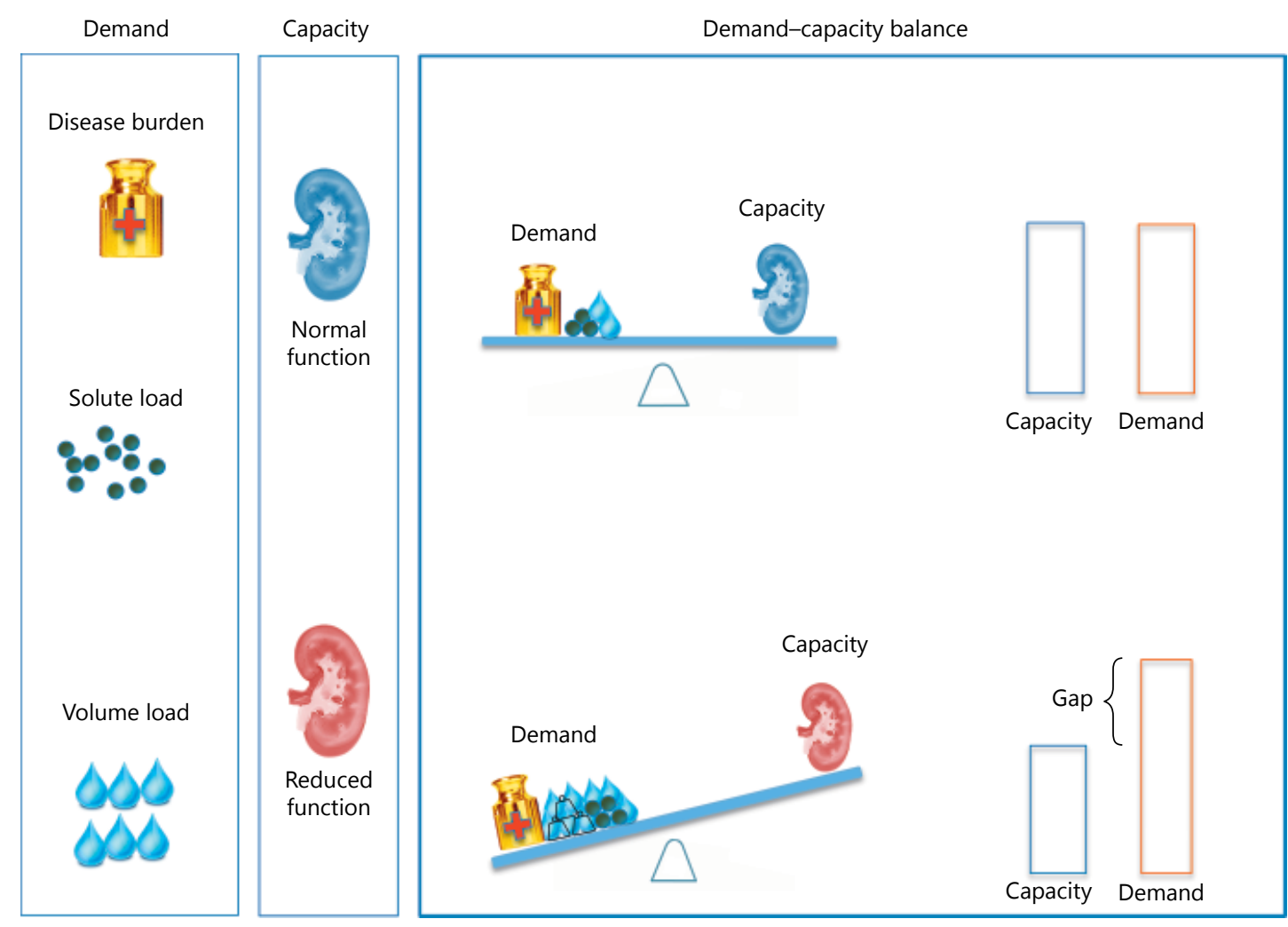

Fig. 1. Demand and capacity - a conceptual model. Reprinted with permission from www.ADQI.org.

and renal capacity exists or is anticipated, RRT should be considered (fig. 1 and 2). Similarly, in case of a decreasing gap, a 'watch and wait strategy' may be justified. For clinical practice, this also means that RRT should be considered at lower stages of AKI if demand is high (table 3). In contrast, RRT may not be necessary even in case of AKI stage 3 if the metabolic and fluid demands are low or decreasing.

\section{Non-AKI Indications for CRRT}

CRRT is an effective method to remove fluid and to achieve a target fluid balance in patients with fluid overload, including those with congestive cardiac failure (CCF) or acute lung injury. Irrespective of whether the consensus criteria for AKI are met, fluid overload should be viewed as an example of demand-capacity imbalance.

In fact, in patients with CCF, fluid overload is a common reason for admission to hospital or ICU. The therapeutic options include pharmacological means and me- chanical fluid removal using extracorporeal techniques [21]. The decision between ultrafiltration (UF) alone versus RRT depends on whether only fluid removal or fluid removal and solute clearance are necessary, Studies investigating the role of UF alone in CCF have shown conflicting results [22-24].

RRT is also effective at removing biologically active substances, including cytokines but there is still insufficient evidence to recommend the routine use of CRRT for the treatment of sepsis. In fact, an RCT comparing early continuous veno-venous hemofiltration $(\mathrm{CVVH})$ versus standard medical treatment in severe sepsis showed that outcome was not improved with CVVH [25]. Finally, in cases of intoxication with a dialyzable/filterable drug or toxin, RRT may also have a role depending on the specific substance to be removed [26].

Consensus statement 1.2: Demand for kidney function is determined by non-renal comorbidities, the severity of the acute disease and solute and fluid burden. 
Table 3. RRT support based on underlying demand and capacity framework*

\begin{tabular}{lll}
\hline Demand & Capacity & Example \\
\hline High & Normal & High catabolic state in patients with normal renal function \\
\hline High & Low & High catabolic state in patient with AKI or CKD \\
\hline Normal & Low & Normal catabolic state in patient with AKI or CKD \\
\hline Low & Low & Malnutrition in patient with AKI or CKD \\
\hline$*$ Adapted from Mehta [19]. & \\
\hline
\end{tabular}

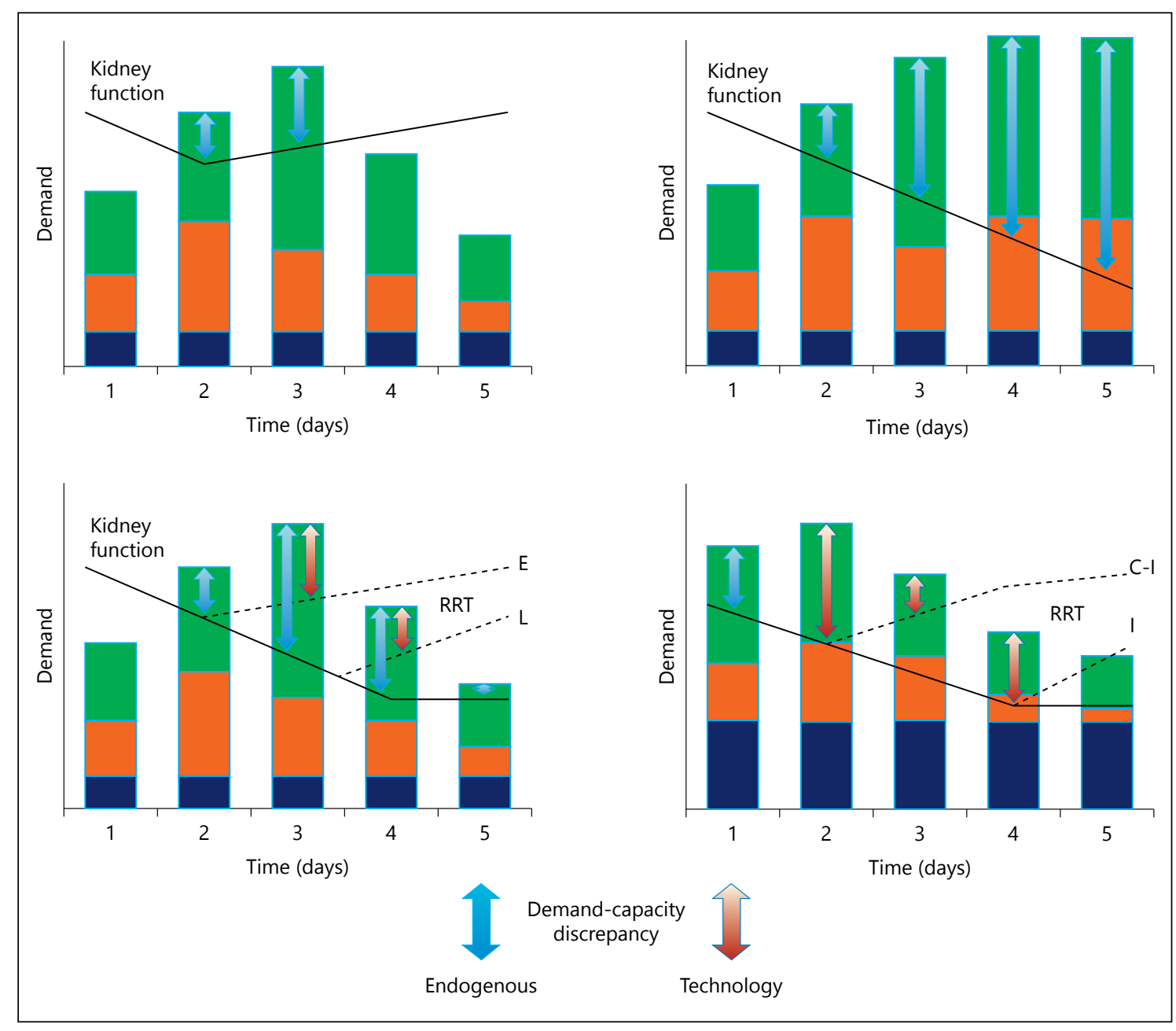

Fig. 2. Shown are 4 patient scenarios. The bars represent total demand including chronic disease (blue), acute illness (orange) and solute/fluid excess (green). The top 2 panels represent no RRT the left illustrates early reversal of AKI and the right shows progressive renal failure and increasing discrepancy between renal function capacity and physiological demands. The 2 bottom panels illustrate the effect of RRT (dashed lines) with (left) early (E) or later (L) initiation and 2 different demand-capacity discrepancy patterns. On the right the patient scenario illustrated is different with high underlying disease burden and either initiation of continuous RRT on day 2 transitioning to IRRT on day 4 (dashed line marked as C-I) or initiation of intermittent therapy on day 4 (I). Reprinted with permission from www.ADQI.org. 
Table 4. Factors affecting metabolic and fluid demand

\begin{tabular}{ll}
\hline Factors & Impact \\
\hline Degree of fluid overload & \\
\hline Solute load (e.g., urea, myoglobin, ammonia) & \\
\hline $\begin{array}{l}\text { Acute severity of illness as defined by any severity of } \\
\text { illness score }\end{array}$ & Increased solute load \\
SOFA & Impaired homeostasis \\
SAPS & Compromised hemodynamics \\
ISS & Increased endovascular permeability \\
EuroSCORE & Macro- and microcirculatory dysfunction \\
CRATE score & $\begin{array}{l}\text { Reduced physiological reserve and } \\
\text { impaired tolerance of fluid overload }\end{array}$ \\
\hline Chronic comorbidities & \\
Chronic heart failure & \\
Chronic lung disease & \\
Age & \\
Chronic liver disease & \\
\hline SAPS = Simplified Acute Physiology Score; ISS = Injury Severity Score; EuroSCORE = score to calculate \\
predicted operative mortality for patients undergoing cardiac surgery; CRATE score = score based on creati- \\
nine, lactic acid, cardiopulmonary bypass time and EuroSCORE.
\end{tabular}

In critically ill patients, the demands on kidney function depend on 3 main factors: (i) severity of the acute disease as measured by degree of inflammation, hemodynamic disturbance, alterations in macro- and microcirculation and metabolic stress; (ii) degree of solute load and fluid accumulation; and (iii) underlying chronic diseases that impact the ability to tolerate volume or solute load (table 4; fig. 1).

Consensus statement 1.3: Total kidney function is measured using a variety of different methods. Changes in kidney function and duration of kidney dysfunction can be anticipated by markers of kidney damage.

Static glomerular function is measured using a variety of different methods. Traditionally, SCr and urine output are used as surrogate markers of renal function [27]. However, they are not renal specific and have important limitations. In particular, SCr changes relatively late after the occurrence of kidney injury and does not indicate the nature or anatomical site of injury. Small absolute changes in SCr may reflect significant kidney damage in patients with normal kidney function but may be physiological in those with pre-existing chronic kidney disease (CKD) [28]. Similarly, oliguria may be an early marker of AKI but may also be physiological (especially if shortlived) and simply reflect hypovolemia rather than injury to the kidney. New biomarkers have emerged that indi- cate renal damage before a rise in SCr or fall in urine output develops. Cystatin $\mathrm{C}$ is an established biomarker of glomerular function in CKD but may not provide additional information when deciding whether to start RRT in patients with AKI $[29,30]$. Several studies have also shown that urinary and plasma NGAL indicated the onset of AKI before SCr [31]. However, like SCr, plasma NGAL is not renal specific and levels can be affected by a wide range of diseases. Urine NGAL may be more specific for renal disease. Some studies have demonstrated that NGAL was predictive of severity of AKI and need for RRT [32]. Urinary insulin-like growth factor binding protein 7 and tissue inhibitor of metalloproteinase 2 are markers of cell cycle arrest and have been shown to predict the development of moderate or severe AKI better than other biomarkers $[32,33]$. However, these markers were developed to predict AKI but not the need for RRT.

Real-time measurement of glomerular function rate (GFR) is not routinely available. However, such monitoring is possible and several investigators are working on the development of rapid, sensitive and affordable techniques to measure GFR in real time in routine clinical practice [34]. Knowing the actual GFR on a moment-tomoment basis might not only detect AKI earlier but also allow better monitoring and possibly, identification of patients in whom RRT should be considered. 
Consensus statement 1.4: The demand-capacity imbalance is dynamic and should be evaluated regularly.

Total demand comprises of chronic disease burden, severity of acute illness and solute and fluid overload. Only chronic comorbidities can be viewed as constant; severity of illness and solute and fluid burden may vary continuously. As such, the demand-capacity balance varies too (fig. 2). In order not to delay RRT in case of an increasing imbalance and to avoid RRT in case of improvement, the demand-capacity imbalance should be evaluated regularly depending on the clinical situation at least once a day.

Consensus statement 1.5: For patients requiring multiple types of organ support, decisions about initiating or withholding RRT should be considered together with other therapies.

Acute RRT is commonly offered to critically ill patients with multi-organ dysfunction. It is extremely effective at correcting uremia and removing excess fluid and should be viewed as a temporary therapy which is often provided in conjunction with other forms of multiorgan support. The potential long-term benefits and risks should be taken into consideration when deciding whether to start RRT, especially in frail and older patients with multiple comorbidities (table 2). Elderly patients represent the fastest growing age group in the general population and are also at high risk of AKI [35-37]. Following an episode of AKI, they have an increased risk of $\mathrm{CKD}$ and end-stage renal disease, long-term morbidity, repeated hospitalizations and mortality $[37,38]$. The median survival of patients aged $\geq 85$ years who received RRT and one or more intensive care procedures (cardiopulmonary resuscitation, feeding tube or mechanical ventilation) was 5 months of which almost 2 months were spent in hospital [39]. Initiation of RRT in this category of patients may prevent acute mortality but at the expense of a decline in functional status and quality of life [40]. In the absence of a survival benefit, important clinical goals such as preservation of dignity, comfort and quality of life should be included in the decisionmaking process [41].

Acute RRT constitutes an integral component of multi-organ support for critically ill patients and has to be considered in conjunction with other forms of lifesustaining therapies. The decision whether to stop, withhold or withdraw RRT should not be made in isolation but take into account the extent of other types of organ support. If the overall treatment goals change and nonrenal life-sustaining therapies are withheld or withdrawn, there is usually little value in initiating or continuing
RRT. Similarly, withholding RRT in situations where fluid and metabolic demand exceed renal capacity will invariably impact the patient's physiological ability to cope and should only be considered if a decision has been made to withhold other forms of organ support too.

Consensus statement 1.6: Once the decision to initiate RRT has been made, the therapy should be started as soon as possible, typically within less than $3 \mathrm{~h}$.

The decision to initiate RRT requires the availability of trained and experienced staff, the insertion of a central venous double lumen catheter and the preparation of a RRT machine. Therefore, invariably, there will be a delay before RRT commences. However, prolonged delays can be avoided by attention to systems organization, and we suggest that treatment with RRT should commence within $3 \mathrm{~h}$ or less after the clinical decision to initiate RRT has been made.

\section{Recommendations for Future Research}

(1) Objective measures (clinical models and/or biomarkers) to quantify demand for kidney function need to be identified.

(2) Measures of renal functional capacity (not just GFR) should be established and tested in the clinical context.

(3) Predictive models (which may include novel diagnostics) should be developed to investigate changes in \#1 and \#2.

(4) There is a need to determine thresholds for demandcapacity imbalance (magnitude and duration) for which initiation of CRRT results in differences in clinical outcomes.

(5) There is a need to investigate differences in patient preferences for various acute invasive organ support therapies.

(6) The time threshold for starting RRT relative to prescription which affects patient-centered outcomes needs to be determined.

What Is the Most Appropriate Therapy to Meet a Demand-Capacity Imbalance for a Specific Patient?

Consensus statement 2.1: Selection of RRT modality depends on the capability/availability of the technology, its inherited risks and the current needs of the patient.

Different RRT modalities provide different capabilities (fig. 3) and thus individual patient needs will be best met by different modalities. This is true across patients and within patients over time. In addition, different machines may provide some but not all modalities [42]. 
Fig. 3. Characteristics and risks of different RRT modalities. PD = Peritoneal dialysis. Reprinted with permission from www. ADQI.org.

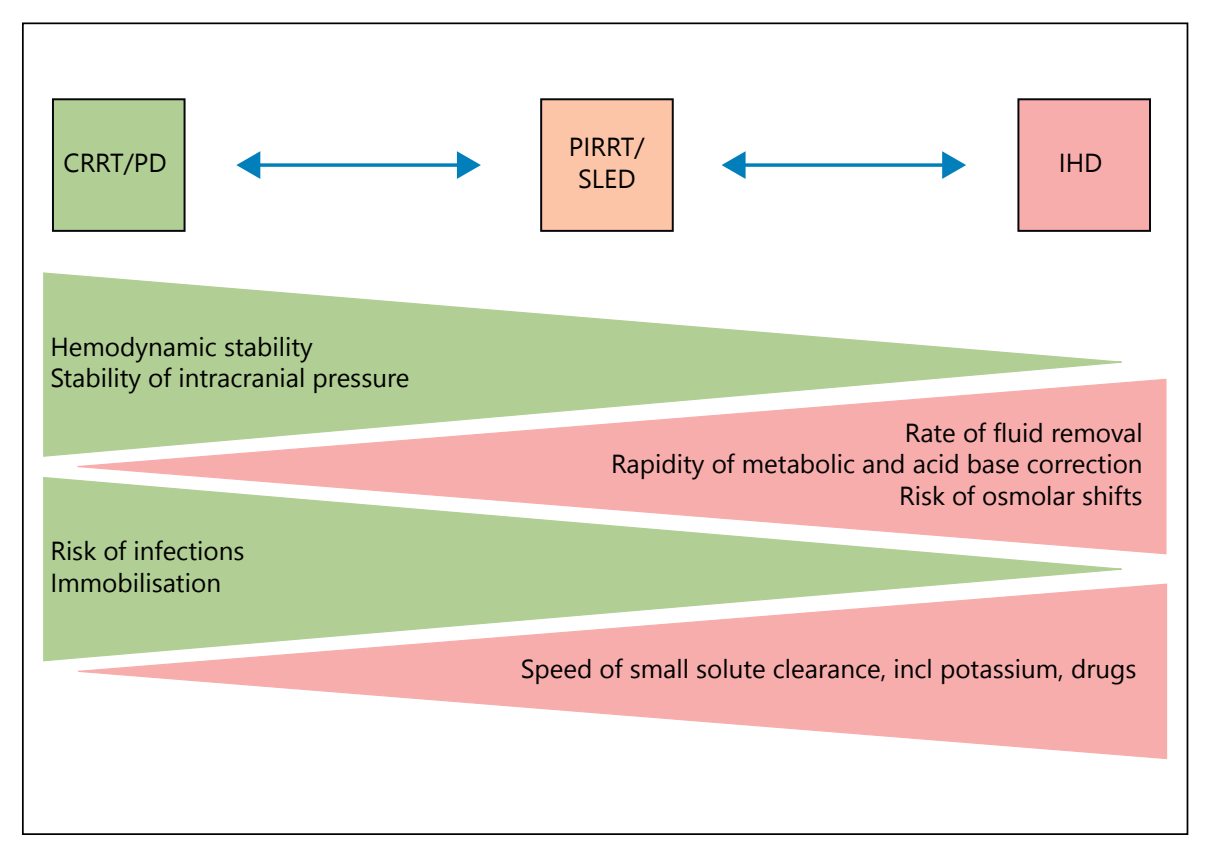

The choice of technology will also be affected by local availability which in turn is determined by regulatory requirements, resources (financial and human) and by the training and expertise of staff. In particular, most facilities will only have a limited number of technologies available and thus the best 'fit' to individual patient needs will be from a small list of machines. Finally, there are inherited risks and burdens associated with each modality (fig. 3 ), which should be considered - see recommendation 2.2.

Consensus statement 2.2: Continuous types of RRT are recommended in situations where shifts in fluid balance and metabolic fluctuations are poorly tolerated. Intermittent and prolonged intermittent types of RRT have a role in situations where rehabilitation or mobilization is the priority, and fluid and metabolic fluctuations can be tolerated.

There are 2 specific situations where preferences for CRRT (over standard intermittent RRT (IRRT)) have been identified. The first is in the setting of intracranial hypertension and/or acute brain injury. We could not find any new evidence addressing this decision since the KDIGO AKI guideline was published in 2012 [27]. KDIGO cited observational data in patients with intracranial pressure monitoring that reported increases in intracranial pressure with IHD [43]. Also, using CT scans to measure brain density, Ronco et al. [44] showed an increase in brain water content after IHD whereas no such changes were observed after CRRT. Thus, we rec- ommend the use of CRRT in patients with, or at increased risk for, cerebral edema.

The second category of patients is the ones with hemodynamic instability. Here, again, the KDIGO guideline noted that many clinicians prefer CRRT in critically ill AKI patients with severe hemodynamic instability because of better hemodynamic tolerance due to the slower fluid removal and the absence of fluid shifts induced by rapid solute removal [27]. Although, a Cochrane metaanalysis could not establish a difference in the number of patients with hemodynamic instability (defined variably) or with hypotension, the mean arterial pressure (MAP) at the end of the treatment was significantly higher and the number of patients requiring escalation of vasopressor therapy was significantly lower with CRRT compared with IHD [45]. Thus, we recommend using CRRT in patients with degrees of hemodynamic instability that exceed the ability to manage the patient safely with more intermittent forms of therapy. We recognize that clinical judgment is necessary to determine the threshold for hemodynamic instability that should prompt the use of CRRT and that local and regional differences in practice exist. The only firm recommendation that can be made is that CRRT is mandatory when RRT is required and more intermittent modalities are not tolerated by the patient.

Both CRRT and IHD achieve adequate metabolic control, and neither modality has been shown to be superior in terms of survival [45-47]. However, some studies suggest that the choice of initial RRT modality may affect 
renal recovery and dialysis dependence after AKI, which has implications for patients, their families and healthcare systems in terms of survival, quality of life and costs [48]. The question whether IHD compared to CRRT is associated with less renal recovery and a higher risk of dialysis dependence was addressed in a meta-analysis of 7 RCTs and 16 observational studies [49]. Pooled analysis of observational studies showed a higher rate of dialysis dependence among survivors who initially received IHD versus CRRT (relative risk (RR) 1.99; 95\% CI 1.53-2.59; $\left.\mathrm{I}^{2}=42 \%\right)$. However, analysis of the RCTs only concluded that there was no difference in rate of dialysis dependence among survivors (RR 1.15; 95\% CI 0.78-1.68; $\mathrm{I}^{2}=0 \%$ ). In a large observational study published following this metaanalysis, Wald et al. [50] reported that CRRT, when compared to IHD as the initial modality of RRT in critically ill adults with AKI, was associated with a lower likelihood of chronic dialysis (hazard ratio 0.75 ; 95\% CI 0.65-0.87). However, this analysis included some centers that only used one modality. In a recent large single-center retrospective cohort study examining adults ( $\geq 18$ years) admitted to ICUs from 2000 to 2008,638 patients received RRT and survived to hospital discharge (353 IHD and 285 CRRT) [51]. Using multivariable analyses, there was no difference in odds for recovery from first RRT modality at 90 or 365 days for patients initially treated with CRRT versus IHD (90 days: OR 1.19, 95\% CI 0.91-1.55, $\mathrm{p}=0.2$; 365 days: OR $0.93,95 \%$ CI $0.72-1.2, \mathrm{p}=0.55$ ).

Consensus statement 2.3: Availability of technologies is determined by local regulations, local resources, including staff, their training/experience and laboratory support and financial constraints. The choice of the technologies that should be made available must balance these issues.

Cost considerations with RRT vary substantially among centers. A study from 2010 identified the relative impact of 4 cost domains (nurse staffing, fluid, anticoagulation and extracorporeal circuit) on overall cost differences and found that the theoretic range of costs were from $\$ 3,629.80 /$ day more with CRRT to $\$ 378.60 /$ day more with IRRT [52]. The median difference in cost between CRRT and IRRT was $\$ 289.60$ (interquartile range 830.8-116.8) per day (greater with CRRT). Costs also vary greatly by region. For instance, a RCT conducted in Germany compared slow efficiency dialysis (SLED) versus CVVH and estimated daily costs of $€ 96.8$ and $€ 258.9$, respectively [53]. Training and experience are both important because centers with very few patients requiring a specific form of support will not be able to maintain competencies with that therapy.

\section{Recommendations for Future Research}

(1) Small precision efficacy studies should be undertaken to compare technologies for their ability to address specific types of demand-capacity imbalance and unintended solute removal.

(2) Large comparative effectiveness trials with patientcentered outcomes should only be undertaken in prespecified patient populations for technologies that have been shown to be efficacious for the specific demand-capacity imbalance being studied.

(3) Hemodynamic measures (e.g., MAP) as surrogates for patient-centered outcomes (e.g., stroke) should be refined and quantified in the clinical context in which acute RRT is performed.

(4) Studies for safety of RRT modalities using surrogates should only be undertaken once they are refined and quantified (see research recommendation 3).

(5) Cost-effectiveness studies of acute RRT should include issues raised in research recommendations 1-4.

\section{How Should RRT Be Integrated into Other Extracorporeal Therapies?}

Consensus statement 3.1: In situations where other extracorporeal therapies are required, continuous RRT is recommended and integrated systems are preferred over parallel systems.

The evolution of extracorporeal life support (ECLS) technology has expanded the therapeutic options for patients with multi-organ failure and those undergoing cardiothoracic surgery or transplantation. The spectrum of ECLS includes veno-arterial (VA) or veno-venous (VV) extracorporeal membrane oxygenation (ECMO), VA or $\mathrm{VV}$ extracorporeal $\mathrm{CO}_{2}$ removal (ECCO2R), ventricular assist devices (VADs), extracorporeal liver assist devices (ELADs), apheresis treatment, including therapeutic plasma exchange (TPE) and renal replacement therapy (RRT). The practice of combining RRT with other forms of ECLS is rapidly increasing. However, high quality evidence-based data are still lacking and clinical practice is variable.

\section{ECLS in Respiratory Failure}

Previous studies in patients treated with VA and VV ECMO demonstrated that the incidence of AKI exceeded $70 \%$ of whom around $50 \%$ needed RRT $[54,55]$. RRT can be provided by introducing the filter into the ECMO circuit (integrated system) or through separate venous access independent of the ECMO circuit (parallel system). It is possible to deliver $\mathrm{CVVH}$, continuous veno-venous 
hemodialysis (CVVHD), continuous veno-venous hemodiafiltration (CVVHDF) and slow continuous ultrafiltration (SCUF) independent of whether the RRT circuit is integrated into the ECMO circuit or separate.

CRRT in patients on ECMO offers more accurate fluid management allowing for more aggressive nutritional support and potentially shorter durations on ECMO [54, 56, 57]. However, the risk of hemolysis appears to be higher in patients receiving RRT and ECMO compared to ECMO alone [54].

The outcome of patients on ECMO and CRRT depends predominantly on the underlying acute illness. A metaanalysis including 43 observational studies with 21,624 patients receiving ECMO suggested that the difference in mortality between patients receiving RRT and those not receiving RRT tended to decrease as the mortality of the group not receiving RRT increased [56]. In patients on RRT, the risk of mortality was greater the longer the initiation of RRT was delayed. In survivors, renal recovery is very good with independence from RRT in $>95 \%$ of patients.

The technique of combining RRT with ECCO2R is similar to that of combining RRT with ECMO with no clear consensus regarding the optimal method. A recent systematic review of 2 RCTs and 12 observational studies concluded that robust data was still lacking, especially since the only 2 RCTs were terminated early for futility [58]. The results of an ongoing active study aimed at evaluating the safety and efficacy of combining ECCO2R and CRRT are awaited (ClinicalTrials.gov; NCT01239966) [59].

\section{ECLS in Cardiac Failure}

Implantable left VADs (LVADs) are increasingly being used as a bridge to transplantation or in the setting of temporary acute cardiac decompensation. Since cardiac contractility is dependent on pre-load, careful attention to fluid removal is crucial. As such, CRRT is the preferred method. Once patients are hemodynamically stable and tolerate fluctuations in fluid status, transition to IRRT may be possible [60].

Peritoneal dialysis is also an option provided the LVAD is implanted in an intra-pericardial or pre-peritoneal location instead of intraperitoneally. In patients receiving prolonged IHD, long-term use of temporary dialysis catheters should be avoided due to the increased risk of blood stream infections.

\section{ECLS in Liver Failure}

In patients with liver disease, the decision between choosing IRRT or CRRT is usually based on the clinical characteristics of the patient. Neither has been demon- strated to be superior to the other but CRRT is better tolerated in those with hemodynamic and metabolic instability. CRRT is definitely indicated in cases of fulminant hepatic failure as it is associated with less fluctuation in intracranial pressure [61].

ELADs can be divided into 2 broad categories: noncell and cell-based systems [62]. Non-cell based systems include molecular adsorbent recirculation systems (MARS) and fractionated plasma separation and adsorption (Prometheus). They offer only detoxification. In contrast, cell-based ELADs combine plasma separation with perfusion of bioreactors filled with human or animal hepatocytes and provide the excretory, synthetic and metabolic functions of the liver $[62,63]$.

The MARS and Prometheus devices deliver hepatic and renal support simultaneously. The MARS system is designed to remove albumin-bound toxins by albumin dialysis as well as providing standard CRRT. The albumin dialysate is then regenerated utilizing an anion exchange resin and active charcoal adsorption. Prometheus employs fractional plasma separation and adsorption with hemodialysis.

Single-pass albumin dialysis with conventional CRRT is feasible too and has been shown to be effective in patients with AKI and high serum bilirubin levels due to liver failure [64]. So far, improved long-term survival with any of these novel ELADs has not been reliably demonstrated but results of ongoing research studies are awaited [65].

\section{RRT and Apheresis Procedures}

Selective apheresis procedures have been developed to target specific molecules, antibodies or cellular elements in a variety of diseases. During TPE, a volume of plasma that is 1-1.5 times the estimated circulating plasma volume is removed and replaced with albumin or clotting products. Newer technologies are available for low-density lipoprotein apheresis, double filtration plasmapheresis, cryofiltration, immunoadsorption procedures, adsorption techniques, extracorporeal photopheresis and leukocyte apheresis [66]. The advantage of the selective apheresis procedures over conventional TPE is preservation of other essential plasma components such as albumin, immunoglobulins and clotting factors. If TPE is necessary in patients already receiving RRT, it can be provided in parallel or, alternatively, via integrated systems where the TPE circuit is attached to the RRT circuit through an extension blood line connected to the RRT return line. There is no clear evidence regarding the optimal technique. 
Fig. 4. Potential pathways following an episode of AKI, including transition of RRT modalities. Reprinted with permission from www.ADQI.org.

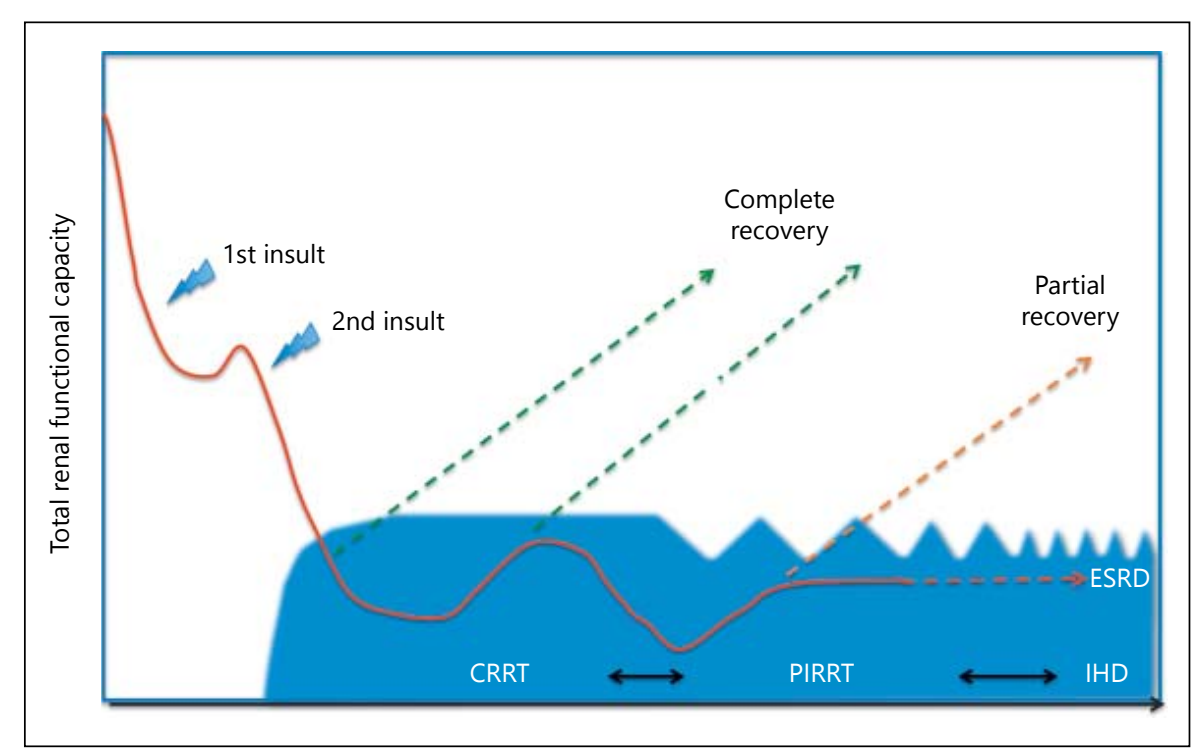

\section{Recommendations for Future Research}

(1) Recognizing that there is increased utilization of multiple organ support therapies, we recommend more research on the risks and benefits of combining these treatments and focusing on safety, efficacy and development of new technology.

When Should Transition of Modalities Be Considered (CRRT, IRRT, Hybrid Therapy)?

Consensus statement 4.1: Transition of modalities should be considered if the demand-capacity imbalance or treatment priorities have changed and can be met better by an alternative technique.

AKI is a disease spectrum ranging from early renal injury to complete renal failure, which can affect patients without any chronic illnesses to critically ill patients with multiple comorbidities and life threatening acute illnesses (sepsis, cardiac surgery, abdominal surgery and neurosurgery). Stratifying patients based on their complexity and individual clinical needs is essential in order to deliver the most appropriate and beneficial RRT modality.

The same principles that apply when considering the most appropriate modality for the first session of RRT should also be applied when evaluating the risks and benefits of switching to another modality (fig. 3 and 4). In addition to IHD and CRRT, hybrid therapies such as 'sustained low efficiency (daily) dialysis' [67], 'extended daily dialysis' (EDD) [68] and 'prolonged IRRT' (PIRRT) have emerged. They combine the hemodynamic stability of CRRT with the advantages of IRRT (mobilization, reha- bilitation, reduced need for anticoagulation, lower financial costs). These modalities utilize either conventional hemodialysis machines adapted to obtain a longer session than traditional IHD [68-70], or conventional CRRT machines for planned treatment sessions for $8-12 \mathrm{~h}$ at higher doses than usual.

Presently there are only 2 studies comparing IHD with PIRRT $[71,72]$. Due to differences in study design (interventional vs. observational), population, severity of illness and the small sample size, it is not possible to make any firm recommendations regarding superiority of either technique [73].

Studies comparing PIRRT with CRRT demonstrated no significant difference in hemodynamic stability and solute clearance $[68,70,74-76]$. In a single-center RCT, Schwenger et al. [53] randomized 232 surgical patients with AKI to SLED versus CRRT. There was no difference in 90-day survival but patients randomized to SLED spent significantly fewer days on mechanical ventilations, required less blood transfusions and had a shorter stay in ICU. A recent meta-analysis including 7 RCTs and $10 \mathrm{ob}-$ servational studies comparing EDD with CRRT in more than 1,200 AKI patients showed no difference in mortality when analyzing RCTs only [77]. However, when analyzing observational studies, there was a significant difference in favor of EDD (RR 0.86; 95\% CI 0.74-1.0; $\mathrm{p}=0.05$ ). Important limitations of all observational studies are the potential risk of allocation and selection bias and lack of stratification according to severity of illness. For example, Wu et al. [70] compared SLED and CRRT in postsurgical patients with severe fluid overload or moderately unstable 
hemodynamics and showed that patients treated with SLED had a higher first MAP post dialysis than those treated with CRRT. However, patients in the CRRT group appeared to be sicker as evidenced by a higher prevalence of sepsis (33 vs. $15.8 \% ; \mathrm{p}=0.065$ ) and need for mechanical ventilation ( 90.5 vs. $73.7 \% ; p=0.046$ ). To date, there is no consensus on important aspects of PIRRT, including frequency, duration and intensity as well as type of RRT machine. Published literature suggests that sessions may range from $6 \mathrm{~h}$ every other day to more than $12 \mathrm{~h}$ daily. This variation in clinical practice has important implications for drug dosing and may account for some differences in results seen in clinical studies.

\section{Recommendations for Future Research}

(1) There is a need to determine the most beneficial method of delivering PIRRT for AKI.

\section{How Should Patients Be Liberated from RRT?}

Consensus statement 5.1: RRT should be discontinued if kidney function has recovered sufficiently to reduce the demand-capacity imbalance (current and expected) to acceptable levels or the overall goals of treatment have changed.

Consensus statement 5.2: To determine sustained recovery of kidney function, we recommend monitoring of urine output and SCr during RRT.

Consensus statement 5.3: For patients requiring multiple types of organ support, decisions about withdrawing RRT should be considered together with other therapies.

Discontinuation of RRT should be considered when the kidneys have regained sufficient function to meet the metabolic and fluid demands placed on them and the previous demand-capacity imbalance is no longer present. The optimal mode of promoting renal recovery and weaning patients off RRT is not known. The administration of furosemide after termination of CRRT does not improve renal function or shorten renal failure [78]. However, furosemide-induced diuresis within $24 \mathrm{~h}$ after cessation of CRRT has been shown to be predictive of eventual renal recovery during hospital stay [79].

The current practice of discontinuing CRRT mostly considers a fall in SCr while on a constant dose of continuous renal support or increases in urine output [80]. Daily urinary urea excretion is used less often but appears to be superior [81]. Results of an observational study conducted in 2000-2002 in 54 ICUs in 23 countries showed that a spontaneous urine output of $400 \mathrm{ml} /$ day was associated with an $80.9 \%$ chance of successful liberation from RRT [80]. In patients receiving diuretics, a urine output of $2,330 \mathrm{ml} /$ day had a positive predictive value of $87.9 \%$. Patients in whom CRRT was discontinued successfully had better outcomes than patients who needed to be restarted on RRT. A retrospective single-center cohort study of 60 patients treated with IRRT for at least 7 days for AKI in an ICU of a French university hospital concluded that a daily urinary urea excretion greater than $1.35 \mathrm{mmol} / \mathrm{kg} / 24 \mathrm{~h}$ was the best marker for weaning ICU patients with AKI from IRRT, followed by urine output greater than $8.5 \mathrm{ml} / \mathrm{kg} / 24 \mathrm{~h}$ [81]. The areas under the receiver operating characteristics curves of daily urinary urea excretion and urine output were 0.96 and 0.86 , respectively.

Acute RRT represents a form of life support. In patients in whom the overall goals of care have shifted towards palliation and end-of-life care and withholding or withdrawal of life sustaining therapies is planned, discontinuation of RRT should also be considered.

\section{Recommendations for Future Research}

(1) It should be determined if different approaches to RRT weaning (e.g., abrupt or gradual or via transition to alternate modalities) affect patient outcomes.

(2) It is necessary to determine whether pharmacologic approaches (e.g., diuretics or growth factors) can modify the success and outcome in the setting of discontinuation of RRT.

(3) It is necessary to determine methods (biomarkers and/or bioinformatics) for predicting the likelihood of renal recovery to determine when to discontinue RRT.

\section{Conclusions}

We suggest that the decision to start, maintain and discontinue CRRT should be individualized based on the demand-capacity concept [20]. Our recommendations may serve to develop the best clinical practice and standards of care for use of CRRT in patients with AKI. Future research is necessary to test and validate our concepts.

\section{Disclosure Statement}

The authors have no conflicts of interest to declare. 


\section{References}

1 Uchino S, Bellomo R, Morimatsu H, et al: Continuous renal replacement therapy: a worldwide practice survey. The beginning and ending supportive therapy for the kidney (B.E.S.T. kidney) investigators. Intensive Care Med 2007;33:1563-1570.

2 RENAL Study Investigators: Renal replacement therapy for acute kidney injury in Australian and New Zealand intensive care units: a practice survey. Crit Care Resusc 2008; 10:225-230.

3 Mehta RL, Letteri JM: Current status of renal replacement therapy for acute renal failure. A survey of US nephrologists. The national kidney foundation council on dialysis. Am J Nephrol 1999;19:377-382.

4 Legrand M, Darmon M, Joannidis M, Payen $D$ : Management of renal replacement therapy in ICU patients: an international survey. Intensive Care Med 2013;39:101-108.

5 Clark E, Wald R, Levin A, et al: Timing the initiation of renal replacement therapy for acute kidney injury in Canadian intensive care units: a multicentre observational study. Can J Anaesth 2012;59:861-870.

6 Hoste EA, Bagshaw SM, Bellomo R, et al: Epidemiology of acute kidney injury in critically ill patients: the multinational AKI-EPI study. Intensive Care Med 2015;41:1411-1423.

7 Kellum JA, Bellomo R, Ronco C: Acute dialysis quality initiative (ADQI): methodology. Int J Artif Organs 2008;31:90-93.

8 Karvellas CJ, Farhat MR, Sajjad I, Mogensen SS, Leung AA, Wald R, et al: A comparison of early versus late initiation of renal replacement therapy in critically ill patients with acute kidney injury: a systematic review and meta-analysis. Crit Care 2011;15:R72.

9 Wierstra BT, Kadri S, Alomar S, Burbano X, Barrisford GW, Kao RL: The impact of 'early' versus 'late' initiation of renal replacement therapy in critical care patients with acute kidney injury: a systematic review and evidence synthesis. Crit Care 2016;20:122.

10 Rewa O, Villeneuve PM, Eurich DT, Stelfox HT, Gibney RT, Hartling L, et al: Quality indicators in continuous renal replacement therapy (CRRT) care in critically ill patients: protocol for a systematic review. Syst Rev 2015;4:102.

11 Wilson FP, Yang W, Machado CA, Mariani LH, Borovskiy Y, Berns JS, Feldman HI: Dialysis versus nondialysis in patients with $\mathrm{AKI}$ : a propensity-matched cohort study. Clin J Am Soc Nephrol 2014;9:673-681.

12 Wald R, Adhikari NK, Smith OM, Weir MA, Pope K, Cohen A, et al: Comparison of standard and accelerated initiation of renal replacement therapy in acute kidney injury. Kidney Int 2015;88:897-904.

13 Zarbock A, Kellum JA, Schmidt C, Van Aken $\mathrm{H}$, Wempe C, Pavenstädt $\mathrm{H}$, et al: Effect of early vs delayed initiation of renal replacement therapy on mortality in critically ill patients with acute kidney injury: the ELAIN randomized clinical trial. JAMA 2016;315: 2190-2199.

14 Gaudry S, Hajage D, Schortgen F, MartinLefevre L, Pons B, Boulet E, et al: Initiation strategies for renal-replacement therapy in the intensive care unit. N Engl J Med 2016; 375:122-133.

15 Singbartl K, Joannidis M: Short-term effects of acute kidney injury. Crit Care Clin 2015;31: 751-762.

16 National Heart, Lung, and Blood Institute Acute Respiratory Distress Syndrome (ARDS) Clinical Trials Network, Wiedemann HP, Wheeler AP, Bernard GR, Thompson BT, Hayden D, deBoisblanc B, Connors AF Jr, Hite RD, Harabin AL: Comparison of two fluid-management strategies in acute lung injury. N Engl J Med 2006;354:2564-2575.

17 Ostermann M, Chang RW: Correlation between parameters at initiation of renal replacement therapy and outcome in patients with acute kidney injury. Crit Care 2009;13: R175.

18 Macedo E, Mehta RL: Continuous dialysis therapies: core curriculum 2016. Am J Kidney Dis 2016;pii:S0272-6386(16)30097-X.

19 Mehta RL: Challenges and pitfalls when implementing renal replacement therapy in the ICU. Crit Care 2015;19(suppl 3):S9.

20 Macedo E, Mehta RL: When should renal replacement therapy be initiated for acute kidney injury? Semin Dial 2011;24:132-137.

21 Rosner $\mathrm{MH}$, Ostermann M, Murugan R, Prowle JR, et al: Indications and management of mechanical fluid removal in critical illness. Br J Anaesth 2014;113:764-771.

22 Costanzo MR, Saltzberg MT, Jessup M, et al: Ultrafiltration is associated with fewer rehospitalizations than continuous diuretic infusion in patients with decompensated heart failure: results from UNLOAD. J Card Fail 2010;16:277-284.

23 Costanzo MR, Negoianu D, Jaski BE, et al: Aquapheresis versus intravenous diuretics and hospitalizations for heart failure. JACC Heart Fail 2016;4:95-105.

24 Bart BA, Goldsmith SR, Lee KL, et al: Ultrafiltration in decompensated heart failure with cardiorenal syndrome. N Engl J Med 2012; 367:2296-2304.

25 Payen D, Mateo J, Cavaillon JM, Fraisse F, Floriot C, Vicaut E; Hemofiltration and Sepsis Group of the Collège National de Réanimation et de Médecine d'Urgence des Hôpitaux extra-Universitaires: Impact of continuous venovenous hemofiltration on organ failure during the early phase of severe sepsis: a randomized controlled trial. Crit Care Med 2009; 37:803-810.

26 Joannidis M, Forni LG: Clinical review: timing of renal replacement therapy. Crit Care $2011 ; 15: 223$

27 Kidney Disease: Improving global outcomes (KDIGO) acute kidney injury work group: KDIGO clinical practice guideline for acute kidney injury. Kidney Int Suppl 2012;2:1138.

28 Palevsky PM, Liu KD, Brophy PD, Chawla LS, Parikh CR, Thakar CV, Tolwani AJ, Waikar SS, Weisbord SD: KDOQI US commentary on the 2012 KDIGO clinical practice guideline for acute kidney injury. Am J Kidney Dis 2013;61:649-672.

29 Spahillari A, Parikh CR, Sint K, et al: Serum cystatin C- versus creatinine-based definitions of acute kidney injury following cardiac surgery: a prospective cohort study. Am J Kidney Dis 2012;60:922-929.

30 Royakkers AA, Korevaar JC, Van Suijlen JD, Hofstra LS, Kuiper MA, Spronk PE, Schultz MJ, Bouman CS: Serum and urine cystatin C are poor biomarkers for acute kidney injury and renal replacement therapy. Intensive Care Med 2011;37:493-501.

31 Hjortrup PB, Haase N, Wetterslev M, Perner A: Clinical review: predictive value of neutrophil gelatinase-associated lipocalin for acute kidney injury in intensive care patients. Crit Care 2013;17:211.

32 Haase M, Bellomo R, Devarajan P, Schlattmann P, Haase-Fielitz A; NGAL Meta-analysis Investigator Group: Accuracy of neutrophil gelatinase-associated lipocalin (NGAL) in diagnosis and prognosis in acute kidney injury: a systematic review and meta-analysis. Am J Kidney Dis 2009;54:1012-1024.

33 Hoste EA, McCullough PA, Kashani K, Chawla LS, Joannidis M, et al: Derivation and validation of cutoffs for clinical use of cell cycle arrest biomarkers. Nephrol Dial Transplant 2014;29:2054-2061.

34 Molitoris BA: Measuring glomerular filtration rate in the intensive care unit: no substitutes please. Crit Care 2013;17:181.

35 Coca SG: Acute kidney injury in elderly persons. Am J Kidney Dis 2010;56:122-131.

36 Del Giudice A, Aucella F: Acute renal failure in the elderly: epidemiology and clinical features. J Nephrol 2012;25(suppl 19):S48-S57.

37 Abdel-Kader K, Palevsky PM: Acute kidney injury in the elderly. Clin Geriatr Med 2009; 25:331-358.

38 Ishani A, Xue JL, Himmelfarb J, Eggers PW, Kimmel PL, Molitoris BA, et al: Acute kidney injury increases risk of ESRD among elderly. J Am Soc Nephrol 2009;20:223-228.

39 Wong SP, Kreuter W, O'Hare AM: Healthcare intensity at initiation of chronic dialysis among older adults. J Am Soc Nephrol 2014; 25:143-149.

40 Kurella Tamura M, Covinsky KE, Chertow GM, Yaffe K, Landefeld CS, McCulloch CE: Functional status of elderly adults before and after initiation of dialysis. N Engl J Med 2009; 361:1539-1547.

41 Fried TR, Tinetti ME, Iannone L, O’Leary JR, Towle V, Van Ness PH: Health outcome prioritization as a tool for decision making among older persons with multiple chronic conditions. Arch Intern Med 2011;171:1854-1856. 
42 Cerdá J, Baldwin I, Honoré PM, Villa G, Kellum J, Ronco C: Role of technology in continuous renal replacement therapy (CRRT) for the management of critically Ill patients: from adoptive technology to precision CRRT. Blood Purif 2016;42:248-265.

43 Davenport A, Will EJ, Davison AM: Effect of renal replacement therapy on patients with combined acute renal and fulminant hepatic failure. Kidney Int Suppl 1993;41:S245-S251.

44 Ronco C, Bellomo R, Brendolan A, Pinna V, La Greca G: Brain density changes during renal replacement in critically ill patients with acute renal failure. Continuous hemofiltration versus intermittent hemodialysis. J Nephrol 1999;12:173-178.

45 Rabindranath K, Adams J, Macleod AM, Muirhead N: Intermittent versus continuous renal replacement therapy for acute renal failure in adults. Cochrane Database Syst Rev 2007;3:CD003773.

46 Bagshaw SM, Berthiaume LR, Delaney A, et al: Continuous versus intermittent renal replacement therapy for critically ill patients with acute kidney injury: a meta-analysis. Crit Care Med 2008;36:610-617.

47 Pannu N, Klarenbach S, Wiebe N, et al: Renal replacement therapy in patients with acute renal failure: a systematic review. JAMA 2008; 299:793-805.

48 Ethgen O, Schneider AG, Bagshaw SM, Bellomo R, Kellum JA: Economics of dialysis dependence following renal replacement therapy for critically ill acute kidney injury patients. Nephrol Dial Transplant 2015;30:54-61.

49 Schneider AG, Bellomo R, Bagshaw SM, Glassford NJ, Lo S, Jun M, Cass A, Gallagher M: Choice of renal replacement therapy modality and dialysis dependence after acute kidney injury: a systematic review and meta-analysis. Intensive Care Med 2013;39:987-997.

50 Wald R, Shariff SZ, Adhikari NK, Bagshaw SM, Burns KE, Friedrich JO, Garg AX, Harel Z, Kitchlu A, Ray JG: The association between renal replacement therapy modality and longterm outcomes among critically ill adults with acute kidney injury: a retrospective cohort study. Crit Care Med 2014;42:868-877.

51 Liang KV, Sileanu FE, Clermont G, Murugan R, Pike F, Palevsky PM, Kellum JA: Modality of RRT and recovery of kidney function after AKI in patients surviving to hospital discharge. Clin J Am Soc Nephrol 2016;11:3038.

52 Srisawat N, Lawsin L, Uchino S, Bellomo R, Kellum JA; BEST Kidney Investigators: Cost of acute renal replacement therapy in the intensive care unit: results from the beginning and ending supportive therapy for the kidney (BEST kidney) study. Crit Care 2010;14:R46.

53 Schwenger V, Weigand MA, Hoffmann O, et al: Sustained low efficiency dialysis using a single-pass batch system in acute kidney injury - a randomized interventional trial: the renal replacement therapy study in intensive care unit patients. Crit Care 2012;16:R140.

54 Chen H, Yu RG, Yin NN, Zhou JX: Combination of extracorporeal membrane oxygenation and continuous renal replacement therapy in critically ill patients: a systematic review. Crit Care 2014;18:675.

55 Antonucci E, Lamanna I, Fagnoul D, et al: The impact of renal failure and renal replacement therapy on outcome during extracorporeal membrane oxygenation therapy. Artif Organs 2016, Epub ahead of print.

56 Han SS, Kim HJ, Lee SJ, et al: Effects of renal replacement therapy in patients receiving extracorporeal membrane oxygenation: a metaanalysis. Ann Thorac Surg 2015;100:14851495.

57 Shekar K, Mullany DV, Thomson B, et al: Extracorporeal life support devices and strategies for management of acute cardiorespiratory failure in adult patients: a comprehensive review. Crit Care 2014;18:219.

58 Fitzgerald M, Millar J, Blackwood B, et al: Extracorporeal carbon dioxide removal for patients with acute respiratory failure secondary to the acute respiratory distress syndrome: a systematic review. Crit Care 2014;18:222.

59 Pulmonary and Renal Support during Acute Respiratory Distress Syndrome. https://clinical trials.gov/ct2/show/NCT01239966?term=NCT 01239966\&rank $=1$.

60 Patel AM, Adeseun GA, Ahmed I, et al: Renal failure in patients with left ventricular assist devices. Clin J Am Soc Nephrol 2013;8:484496.

61 Davenport A: Continuous renal replacement therapies in patients with liver disease. Semin Dial 2009;22:169-172.

62 Nadim MK, Kellum JA, Davenport A, et al: Hepatorenal syndrome: the 8th international consensus conference of the acute dialysis quality initiative (ADQI) group. Crit Care 2012;16:R23

63 Gonwa TA, Wadei HM: The challenges of providing renal replacement therapy in decompensated liver cirrhosis. Blood Purif 2012;33:144-148.

64 Chawla LS, Georgescu F, Abell B, et al: Modification of continuous venovenous hemodiafiltration with single-pass albumin dialysate allows for removal of serum bilirubin. Am J Kidney Dis 2005;45:e51-e56.

65 Bañares R, Nevens F, Larsen FS, et al: Extracorporeal albumin dialysis with the molecular adsorbent recirculating system in acute-onchronic liver failure: the RELIEF trial. Hepatology 2013;57:1153-1162.

66 Sanchez AP, Cunard R, Ward DM: The selective therapeutic apheresis procedures. J Clin Apher 2013;28:20-29.

67 Marshall MR, Golper TA, Shaver MJ, et al: Sustained low-efficiency dialysis for critically ill patients requiring renal replacement therapy. Kidney Int 2001;60:777-785.

68 Kumar VA, Craig M, Depner TA, et al: Extended daily dialysis: a new approach to renal replacement for acute renal failure in the intensive care unit. Am J Kidney Dis 2000;36: 294-300.

69 Berbece AN, Richardson RM: Sustained lowefficiency dialysis in the ICU: cost, anticoagulation, and solute removal. Kidney Int 2006; 70:963-968.

$70 \mathrm{Wu} \mathrm{VC}$, Wang CH, Wang WJ, et al: Sustained low-efficiency dialysis versus continuous veno-venous hemofiltration for postsurgical acute renal failure. Am J Surg 2010;199:466476

71 Khanal N, Marshall MR, Ma TM, et al: Comparison of outcomes by modality for critically ill patients requiring renal replacement therapy: a single-centre cohort study adjusting for time-varying illness severity and modality exposure. Anaesth Intensive Care 2012;40:260268

72 Kumar N, Ahlawat RS: Extended daily dialysis in acute renal failure: a new therapeutic approach. Iran J Kidney Dis 2007;1:63-72.

73 Neves JB, Rodrigues FB, Castelão M, et al: Extended daily dialysis versus intermittent hemodialysis for acute kidney injury: a systematic review. J Crit Care 2016;33:271-273.

74 Kumar VA, Yeun JY, Depner TA, et al: Extended daily dialysis vs. continuous hemodialysis for ICU patients with acute renal failure: a two-year single center report. Int J Artif Organs 2004;27:371-379.

75 Kielstein JT, Kretschmer U, Ernst T, et al: Efficacy and cardiovascular tolerability of extended dialysis in critically ill patients: a randomized controlled study. Am J Kidney Dis 2004;43:342-349.

76 Marshall MR, Ma T, Galler D, et al: Sustained low-efficiency daily diafiltration (SLEDD-f) for critically ill patients requiring renal replacement therapy: towards an adequate therapy. Nephrol Dial Transplant 2004;19:877-884.

77 Zhang L, Yang J, Eastwood GM, et al: Extended daily dialysis versus continuous renal replacement therapy for acute kidney injury: a meta-analysis. Am J Kidney Dis 2015;66:322_ 330.

78 van der Voort PH, Boerma EC, Koopmans M, et al: Furosemide does not improve renal recovery after hemofiltration for acute renal failure in critically ill patients: a double blind randomized controlled trial. Crit Care Med 2009;37:533-538.

79 Van der Voort PH, Boerma EC, Pickkers P: The furosemide stress test to predict renal function after continuous renal replacement therapy. Crit Care 2014;18:429.

80 Uchino S, Bellomo R, Morimatsu H, et al: Discontinuation of continuous renal replacement therapy: a post hoc analysis of a prospective multicenter observational study. Crit Care Med 2009;37:2576-2582.

81 Aniort J, Ait Hssain A, Pereira B, et al: Daily urinary urea excretion to guide intermittent hemodialysis weaning in critically ill patients. Crit Care 2016;20:43. 